\title{
К ВОПРОСУ ГИДРОДИНАМИЧЕСКОГО МОДЕЛИРОВАНИЯ ПРОЦЕССОВ ВОСХОДЯЩЕЙ МИГРАЦИИ РЕОЛОГИЧЕСКИ СЛОЖНЫХ НЕОДНОРОДНЫХ ФЛЮИДОВ ИЗ ГЛУБИННЫХ ПОДЗЕМНЫХ КОЛЛЕКТОРОВ
}

\author{
Мельник Игорь Анатольевич 1 , \\ melnik@tpu.ru
}

\author{
Харламов Сергей Николаевич1, \\ kharsn@mail.ru \\ 1 Национальный исследовательский Томский политехнический университет,
Россия, 634050, г. Томск, пр. Ленина, 30.
}

\begin{abstract}
Актуальность вызвана необходимостью разработок новых математических моделей, методов, экспериментальных подходов при исследовании проблем миграции глубинных углеводородных фрлюидов из подземных коллекторов нефртегазового бассейна. Цель: разработать универсальную фризическую и математическую модель детального прогноза миграции по ограниченному пространству подземного коллектора произвольной геометрии; выдать рекомендации в практику исследования задач гидродинамики и тепломассообмена в данных условиях; сопоставить относительные величины сил, являющихся причиной миграционных процессов глубинных фрлюидов в вертикальном и латеральном направлениях.

Методы исследования базируются на общих принципах механики сплошных гомогенных и гетерогенных сред, гидродинамики и тепломассопереноса.

Результаты. Представлены данные анализа эфрфектов, сопровождающих гидрогазодинамику и тепломассоперенос при течениях гомогенных и гетерогенных реологически простых и сложных вязких смесей в полях действия внешних сил из подземных областей с произвольной геометрией щелевого и порового пространства. Проведен анализ корректности положений физического моделирования задачи о миграции подземных углеводородов и установления определяющих механизмов их подъема. Установлено, что восходящая миграция формируется под определяющим влиянием в результирующей силе: выталкивающей силы $\left(\boldsymbol{F}_{B}\right)$, учитывающей особенности изменений полей термодинамических параметров (состава (сі), температуры $(T)$, давления $(P)) ;$ силы тяжести $\left(\boldsymbol{F}_{\boldsymbol{G}}\right) ;$ фразовых явлений и процессов на границах исследуемого объема текущей углеводородной среды, а также силы градиента давления $\left(\boldsymbol{F}_{P R}\right)$, поведение которой осложняется распределением всего спектра внешних и внутренних сил в сопряженной системе. Даны заключения о деталях моделирования движения фллюида, формулировках определяющих чисел и критериев в рамках теории подобия рассматриваемых задач, полезных для уяснения и уточнения сути эфрфектов, процессов, сил, механизмов, сопровождающих миграцию. Результаты могут быть использованы для прогноза миграции, особенно в условиях, осложненных: аномальными фразовыми и реологическими мелкомасштабными дифффузионными изменениями состава; сопряженньм тепломассопереносом между породой и смесью; нестабильностью режимов в локальных областях течений фрлюида при произвольной термодинамической и геометрической конфицурациях подземного коллектора.
\end{abstract}

Ключевые слова:

Флюид, смеси, миграция, моделирование, гидродинамика, тепломассообмен, реология, структура, массовые силы.

\section{Введение}

Исследования закономерностей формирования углеводородных (УВ) залежей в упруго-деформируемых средах, поиска месторождений полезных ископаемых, управления процессами их добычи связаны с необходимостью математического моделирования гидрогазодинамики и сопряженного тепломассообмена в гомогенных и гетерогенных средах со сложными реологическими свойствами, составом, структурой, эндогенными процессами, определяющими эволюцию фильтрационных и низкорейнольдсовых течений многофазных флюидов в проницаемых зонах. Решение таких нетривиальных и комплексных задач крайне важно для понимания и уяснения механизмов формирования условий направленного пространственного движения глубинных флюидов, а также разработки комплексных критериев прогноза локальных областей их залегания [1].

Стоит отметить, что, начиная с работы [2], особое практическое значение отмеченных проблем приняло научное направление по математическому моделированию течений реологически сложных вязких тече- ний УВ смесей с тепло- и массопереносом в полях действия внешних сил. Имеющиеся публикации по проблемам глубинной нефти, например, [3-6], указывают на интерес к вопросам:

1) влияния плотностной неоднородности, направления скоростей, интенсивности движения флюидов из больших глубин нефтегазового бассейна при детальном анализе проблем моделирования процессов переноса в диффузионном приближении с учетом перекрестных эффектов, фильтрации многокомпонентных инертных и химически реагирующих смесей, распространений ударных волн и т. п.;

2) термобарической неоднородности $[5,6]$, разработки комплексного подхода исследования интенсивности движения флюидов с вязкостноинерционно-гравитационными эффектами;

3) формирования УВ залежей в упругодеформируемых средах с криволинейной поверхностью, осложненных влиянием сопряженных процессов в системах «внешняя среда - флюид»;

4) гидродинамики и тепломассообмена (ТМО) при фазовых эффектах и переходах в системе «флюид - 
порода - компонентный состав», спектра имеющихся пульсаций термодинамических параметров и их корреляционных моментов в рамках моделей химически реагирующей сплошной среды, обусловленных процессами в глубинных магматических очагах и разломах земной коры $[7,8]$.

Причем вопросы первой группы - моделирования потоков и термодинамических движущих сил различной природы - наиболее актуальны для приложений, т. к. составляют основу практически всех диффузионных процессов в гидро- и геодинамике, тепломассопереносе, описываемых в рамках термодинамики неравновесных процессов. Фундаментальные положения, заключения и обобщения результатов этой группы важны в исследованиях закономерностей процессов переноса массы, тепла и импульса в системaх, осложненных: действием вязких эффектов; переходами тепловой, вихревой и энтропийной природы; интенсивностью вторичных течений; скрещивающими явлениями; активным и консервативным влиянием массовых сил на локальную структуру; процессами самоорганизации вещества при движении глубинных флюидов со специфическими рео- и теплофизическими свойствами. Кроме того, стоит отметить, что при моделировании геологических и геофизических процессов следует учитывать межфазные механизмы переноса флюида с течениями пластовых вод в пористых и гранулированных средах, которые осложнены фильтрационными и конвективными явлениями. Очевидно, что в реальных условиях основной объект исследования (процессы гидрогазодинамики и ТМО при течении как гомогенных, так и гетерогенных флюидов в глубоких горизонтах) весьма сложен и его физическое моделирование должно быть структурировано.

Цель данной работы состоит в построении комплексных термодинамически согласованных физикоматематических моделей течения реологически сложных гомогенных и гетерогенных углеводородных сред. Причем повышенное внимание уделяется изучению локальных особенностей и эффектов, определяющих эволюцию структуры флюида в поровом пространстве.

Решение указанной задачи требует: определения спектра эффектов и сил, сопровождающих стационарное/нестационарное,

изотермическое/неизотермическое движение флюида как реологически сложной вязкой гомогенной/гетерогенной (двухфазной) смеси в полях действия внешних сил в замкнутых областях, представляющих коллекторы с упруго-деформируемой/недеформируемой поверхностью; верификации метода расчета и валидации математической модели по имеющимся данным для гомогенной (гетерогенной) среды.

Рассмотрим пример результатов глубинной миграции кислых флюидов, обуславливающих вторичные геохимические преобразования аллотигенных минералов в песчаных породах на Тамбейском нефтегазовом месторождении полуострова Ямал. Так, на рис. 1 изображено распределение (в пределах меловых отложений ПК1-БЯ17) интенсивности вторичной каолинитизации, определяемой по инновационной технологии на базе данных геофизических исследований скважин [9]. Заметно то, что с повышением глубины залегания пластов интенсивность процесса наложенного эпигенеза (каолинитизации) уменьшается. Это является подтверждением вертикальной миграции глубинных кислых гидротерм, что, в свою очередь, возможно только в случае присутствия глубинных каналов миграции, т. е. разломов растяжения, окаймляющих их трещин и ослабленных пористых сред. Интенсивность процесса наложенного эпигенеза в коллекторах-песчаниках при фильтрации глубинных флюидов вверх еще сильно зависит от качества покрышек, флюидоупоров, встречаемых на пути их миграции. При мощных и слабопроницаемых покрышках, с соответствующей длительностью времени существования нераскрытых ловушек, интенсивность вторичных преобразований нижних песчаных пород будет достаточно высокой вследствие повышенной продолжительности геохимических преобразований. Это наглядно демонстрируют данные рис. 1, свидетельствующие о том, что присутствие вверху покрышек (R1; R4; ТП10) аномально увеличивает интенсивность вторичной каолинитизации в нижних песчаных пластах.

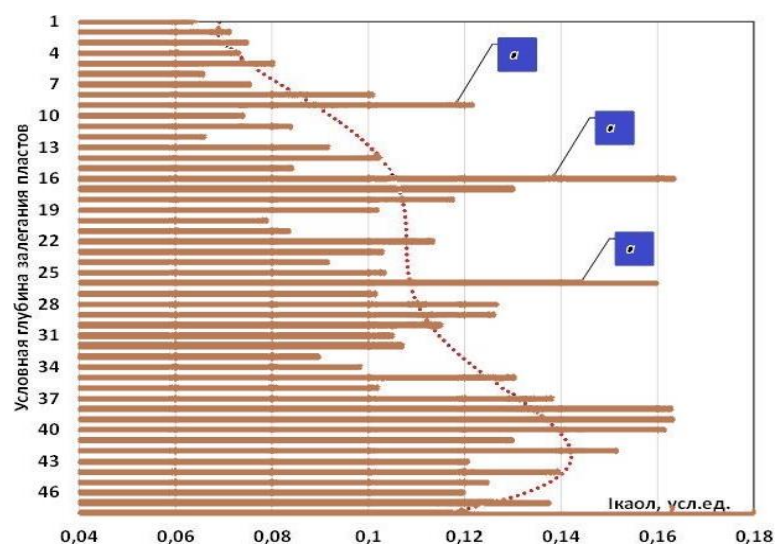

Рис. 1. Распределение по условной глубине залегания интенсивности вторичной каолинитизации, усреднённой по песчаным пластам 38-ми скважин Тамбейского месторождения (n-в Ямал), где а - проявление аномально высокой интенсивности

Fig. 1. Distribution by the conditional depth of occurrence of secondary kaolinitization intensity averaged by sandy formations of 38 wells of the Tambeysky field (Yamal half Island), where a is a manifestation of abnormally high intensity

Таким образом, интенсивность вторичной каолинитизации служит показателем миграции флюидов и может быть косвенным параметром, подтверждающим факт вертикальной флюидомиграции, обусловленной проявлением результирующей силы, включающей градиент литостатического давления, вектор которого направлен вверх.

\section{Допущения к физической модели движения фрлюидов}

Интуитивные представления о процессах и механизмах переноса, сопутствующих и регулирующих 
движение флюида в глубинных коллекторах, позволяют утверждать, что деформационные и межфазные явления в породе и флюиде отличаются многомерностью, а также сильной многопараметрической зависимостью термодинамических параметров от характера и интенсивности изменений полей внешних и внутренних сил. Следует ожидать, что в упругодеформируемой сопряженной системе «флюидпорода» процессы сжатия и разлома будут усложнять внутрифазные мелкомасштабные процессы переноса скаляра (тепла и массы) и генерировать дополнительные термо-, баро-, дино- и концентрационные диффузионные эффекты. Пренебрежение этими процессами без реальных оценок величин соответствующего вклада в общий баланс изменений массы индивидуальных компонент в упруго-термических режимах фильтрации может приводить к серьезным ошибкам в прогнозах движений и пространственного распределения фаз (компонент) флюида в коллекторе.

Краткий анализ проблем, представленных, например, в [1-9], показывает, что в настоящее время резко вырос практический интерес к гидродинамическому и геохимическому моделированию глубинных литосферных флюидных систем, в большинстве своем требующих фундаментального исследования закономерностей движения УВ сред, эффектов движения частиц примеси на динамику потока в глубоких горизонтах. Также ценными для практики выступают вопросы гидродинамического моделирования указанных геодинамических процессов при эндогенном (импульсном) воздействии в рамках использования многоскоростных (в частности, двухскоростных) моделей. Их численные результаты представляются перспективными в исследовании пространственновременных закономерностей изменений термодинамических параметров флюида, геохимическом анализе взаимодействий системы «флюид-порода» в литосферных проницаемых зонах, выяснении комбинированного/раздельного характера восходящей и/или нисходящей миграции подземных вод и углеводородов в глубинных нефтегазоносных бассейнах. Эти результаты могут быть получены на основе детального комплексного напряженно-деформируемого и теплои гидродинамического моделирования параметров состояния сопряженной системы.

При построении физической модели течения флюида, оценок восходящего или нисходящего движений составляющих его фаз/компонентов в глубинных горизонтах необходимо обратиться к следующим основным положениям.

\section{Условная схема характерных сил и их моделирование}

Выяснение общей сути явлений, сопровождающих, организующих и управляющих восходящим/нисходящим движением флюида в глубинных областях нефтегазоносных бассейнов требует: оценки характера и особенностей взаимодействующих сил в окрестности точки приложения к элементу объема смеси, представляющей флюид (частица-жидкость, жидкостьжидкость и т. п.); представлений о режимах, механизмах процессов переноса в такой системе, опреде- ляемых свойствами флюида, внешней среды, деталями структурного состава границы, формы поверхности взаимодействия и полной геометрии области исследования. Эти данные важны и составляют основу формулировки условий однозначности физикоматематической модели при ее численной реализации.

Без нарушений общности заключений о гидродинамике и ТМО в сопряженной системе, с целью простоты формулировки картины сил и уяснения процессов при течении флюида в поровом пространстве с: характерными линейными продольным $\left(L_{x}\right)$, поперечным $\left(L_{y}\right)$, и азимутальным $\left(L_{z}\right)$ масштабами; криволинейной внутренней границей, предположим, что коллектор имеет поры, щели, расположенные под углом $\boldsymbol{a}$ к горизонту, согласно схеме на рис. 2.

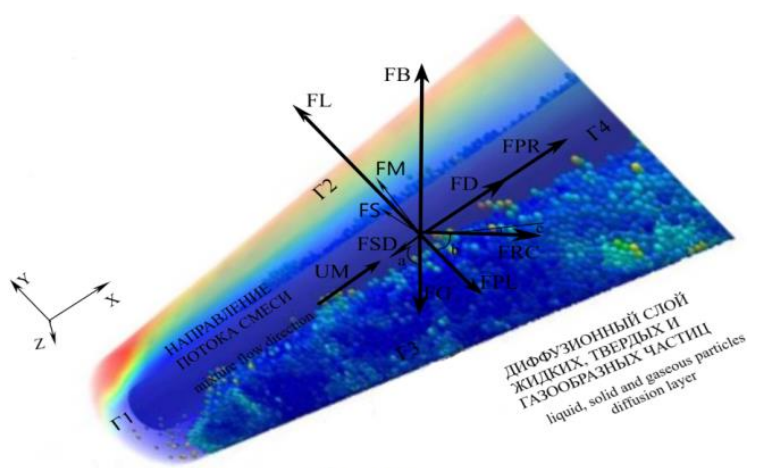

Pис. 2. Условная схема сил, действующих на флюид с гетерогенной структурой, определяюших возникновение равнодействующего вектора (результирующей силь) и прочессов переноса в пористой протяженной области координатного пространства (коллектора НГБ) при восходяuуей миграичии

Fig. 2. Conditional diagram of forces acting on a fluid with a heterogeneous structure, determining the occurrence of a total vector (resultant force) and transfer processes in the porous extended area of the coordinate space (collector) during upward migration

В обозначениях учитывается: $X, Y, Z$ - соответственно осевое, нормальное и азимутальное направления восходящего потока в ортогональной декартовой системе координат $(o X Y Z) ; \mathbf{F}_{\mathrm{D}},[\mathrm{N}]$ - сила сопротивления тяжелых (жидких/твердых) частиц при движении с их характерной локальной скоростью, $\mathrm{U}_{\mathrm{L}}$, $[\mathrm{m} / \mathrm{s}] ; \mathbf{F}_{\mathrm{SD}},[\mathrm{N}]$ - полная сила сопротивления частиц флюида восходящему потоку со скоростью, $\mathrm{U}_{\mathrm{M}},[\mathrm{m} / \mathrm{s}]$; $\mathbf{F}_{\mathrm{B}},[\mathrm{N}]$ - сила плавучести (выталкивающая или архимедова); $\mathbf{F}_{\mathrm{L}},[\mathrm{N}]$ - подъемная сила; $\mathbf{F}_{\mathrm{M}},[\mathrm{N}]$ - дополнительная подъемная сила (Магнуса); $\mathbf{F}_{\mathrm{S}},[\mathrm{N}]$ - дополнительная подъемная сила (Саффмена); $\mathbf{F}_{\mathrm{G}},[\mathrm{N}]$ - сила тяжести; $\mathbf{F}_{\mathrm{PL}},[\mathrm{N}]$ - сила реологических эффектов частиц смеси; $\mathbf{F}_{\mathrm{RC}},[\mathrm{N}]$ - сила реакции взаимодействующих твердых недеформируемых частиц; $\mathbf{F}_{\mathrm{PR}},[\mathrm{N}]-$ сила градиента давления; $\mathbf{U}_{\mathrm{M}},[\mathrm{m} / \mathrm{s}]$ - характерная среднемассовая скорость смеси; $\boldsymbol{a}$ - угол поры, щели коллектора при отклонении от вертикальной плоскости, $\left[{ }^{\circ}\right] ; \boldsymbol{b}$ - угол слоя частиц капельной и твердой фазы, $\left[^{\circ}\right] ; \boldsymbol{c}$ - угол контакта между частицами диффузионного слоя, $\left[^{\circ}\right] ; \Gamma i,(i=1-4)$ - соответственно границы 
области течения смеси: входной зоны (1), верхней (2) и нижней (3) зоны, образующих поверхности поры (щели), зоны выхода (4) потока. Также цветом выделены характерные области динамики частиц смеси в условиях действия слабых (темный фон) и интенсивных (фон светло-красных оттенков) восходящих токов частиц смеси по пространству пор (узких щелей коллектора) с учетом особенностей расслоения структуры диффузионного слоя на области тяжелых частиц (гранул), капельной (холодный цвет) и газообразной (яркий фон) составляющих гетерогенной смеси.

Заметим, что в случае интенсификации процессов переноса, обусловленных потерей устойчивости из-за наличия переходов вихревой, тепловой природы, в локальных областях могут формироваться дополнительные силы, интенсифицирующие восходящую миграцию (Саффмена, Магнуса). Так, например, сила Саффмена будет определятся неоднородностью профиля скорости несущей среды в условиях различий относительных скоростей обтекания частиц флюида с различных сторон и действия перепада давления. В таких процессах движение частицы будет ориентировано в сторону пониженного давления. Тогда как сила Магнуса будет формироваться вращательным движением частиц, в силу того, что при движении потока смеси частицы приобретают вращательный импульс за счет соударений друг с другом и с границей поверхности коллектора. Поэтому, вращаясь, твердая частица будет способна увлекать за собой частицы несущей среды (например, газ).

Также для уяснения сути процессов, сопровождающих течение, представленное на рис. 2, важно отметить, что скорость $\mathbf{U}_{\mathrm{M}}$ весьма чувствительна к условиям, процессам, внутренним/внешним силам, эффектам в открытой термодинамической системе «флюид - граница раздела - порода». Твердые частицы смеси, капли нефти, воды коагулируются в локальных областях щелевого пространства, причем эти зоны не обязательно соответствуют пристеночной части, а формируются эффектами взаимодействия сил и процессов. Предполагая, что неизотермические процессы в системе, обусловленные вероятностью разломов, эндогенных, химических, электродинамических явлений и т. П., вносят неустойчивость в динамику и ТМП, допускается существование спектра сил, распределенных в соответствии с рис. 2. В таких условиях видно, что восходящая миграция частиц флюида может возникнуть в результате взаимодействия спектра сил, вызывающих изменение состава среды. Разность плотностей может определяться переносом тепловой энергии и ее механизмов. Также данные рис. 2 показывают, что результирующее течение определяется эффектами и особенностями распределений внутренних и внешних сил, конфигурацией границ и формой взаимодействующих частиц друг с другом. Важно отметить, что вследствие нелинейности изменений теплофизических свойств отдельных частиц и смеси в целом даже в локальных областях со сравнительно слабыми скоростями движения фракций (для которых инерционные, вязкие, диффузионные и гравитационные эффекты будут од- ного порядка) картины изменений теплогидродинамических и концентрационных полей сильно коррелированы между собой. Следует учитывать, что в рассматриваемом случае течение флюида чувствительно к «тонким» прямым и перекрестным механизмам, которые возникают в гомогенной и гетерогенной неизотермических средах. И для определения изменений фазовых состояний, переноса скаляра (тепла, массы), диффузии одного или нескольких различных химических веществ (компонентов/фаз) целесообразно обращение к формулировке процессов дино-, баро-, термо-, концентрационной диффузии согласно общим принципам термодинамики [10, 11], аксиоматическим линейным связям потоков с термодинамическими движущими силами по теории Онзагера, принципам Ле-Шателье-Брауна и Кюри. Кроме того, также требуют внимания оценки режимов течений, вызванных выталкивающей силой, начиная с малых масштабов, поскольку следует ожидать появления возможной нестабильности состава смеси, неустойчивостей типа вихревой и тепловой природы при ее движении.

Таким образом, следуя данным рис. 2, задача установления миграции смеси со стратификацией плотности в термодинамической системе «флюидпорода» под воздействием спектра локальных движений и возмущений, вызванных выталкивающей силой, заставляет обращаться к моделям, непосредственно учитывающим особенности течения реологически сложной вязкой среды.

\section{Детали оценки результирующей силы}

Хорошо известно, что основу формулировки процессов течения однородного флюида составляют уравнения движения (1), (2), которые в направлениях интенсивного переноса $(x, y)$ декартовой системы координат в своей общей форме, согласно рис. 2 , имеют вид [12-14]:

$$
\begin{gathered}
\rho\left(\frac{\partial V_{x}}{\partial t}+(\bar{V} \cdot \nabla) V_{x}\right)=F_{\Sigma x} ; \\
\rho\left(\frac{\partial V_{y}}{\partial t}+(\bar{V} \cdot \nabla) V_{y}\right)=F_{\Sigma y}, \\
F_{\Sigma x}=F_{L x}+F_{B x}+F_{M x}+F_{S x}+F_{S D x}+ \\
+F_{G x}+F_{P L x}+F_{R C x}+F_{D x}+F_{P R x} ; \\
F_{\Sigma y}=F_{L y}+F_{B y}+F_{M y}+F_{S y}+F_{S D y}+ \\
+F_{G y}+F_{P L y}+F_{R C y}+F_{D y}+F_{P R y} .
\end{gathered}
$$

При формулировке оценок указанных сил полезны замечания, суть которых состоит в следующем.

Сила плавучести, $\boldsymbol{F}_{B}$

С целью детализации и уяснения эффектов, обусловленных выталкивающей силой, как одной из определяющих восходящую миграцию, и простоты заключений, оценок ее влияния на течение, осложненное локальным тепломассопереносом (например, $[15,16])$, целесообразно воспользоваться отдельными допущениями (I, II). 
I. Принимаем, что силы инерции близки по порядку выталкивающей силе и определяем ее поведение связью: $(\bar{V} \cdot \nabla) \bar{V} \approx \bar{F}_{\text {в }}$.

II. Допускаем, что движение флюида в изотропной/анизотропной пористой среде происходит в условиях, когда силы инерции пренебрежимо малы.

В обоих случаях из-за высоких значений $\nabla T, \nabla P$, $\nabla \rho$ в физическом процессе в глубинных горизонтах при оценках порядков сил целесообразно учитывать нелинейность явлений в процессах переноса тепла, массы и импульса, осложняющих процесс моделирования [17]. Поэтому при полной формулировке вопроса оценки гидродинамических процессов и сил, определяющих восходящую миграцию флюида, следует привлекать полные уравнения закона сохранения импульса (3) гомогенных/гетерогенных, однородных/неоднородных, ньютоновских/неньютоновских сред (например, [11, 14, 18-22]), которые в своей простой форме имеют вид [12, 13]:

$$
\rho\left(\frac{\partial \bar{V}}{\partial t}+(\bar{V} \cdot \nabla) \bar{V}\right)=\bar{F}_{\Sigma}=\bar{F}_{\Sigma \mathrm{M}}+\bar{F}_{\Sigma \mathrm{m}}=\bar{F}_{\Sigma \mathrm{M}}+D I V \stackrel{\bar{P}}{.}
$$

Здесь $\bar{F}_{\Sigma \mathrm{m}}, \overline{\bar{P}}$ - вектор результирующей внешней силы, тензор вязких напряжений соответственно.

Тогда в рамках допущения I при формулировке неизвестных параметров и определении замкнутости условий задачи целесообразно:

- обращаться к условиям динамического, теплового и диффузионного подобия. Однако в этом случае решение осложняется тем, что чем больше число отдельных сил, требующих описания течения, тем меныше имеется возможностей для независимого выбора параметров исследуемых динамически подобных моделей;

- устанавливать значения определяющих критериев подобия с учетом существенности вклада отдельных сил в формирование общей картины течения флюида в системе.

Это позволяет общую систему определяющих безразмерных уравнений (4)-(6), описывающих гидродинамику и теплообмен при движении однородного химически инертного флюида с постоянными физическими свойствами в доминирующем продольном направлении (для простоты изложения) представить в виде [23, 24]:

$$
\begin{gathered}
\frac{\partial \theta}{\partial \mathrm{Fo}}+\operatorname{Pe}(\bar{V} \cdot \nabla) \theta=\nabla^{2} \theta, \\
\frac{\partial V_{x}}{\partial \mathrm{Zh}}+\operatorname{Re}(\bar{V} \cdot \nabla) V_{x}=-\frac{\mathrm{Gr}}{\operatorname{Re}} \theta \cos a-\frac{\partial}{\partial x}(\mathrm{Eu} \mathrm{Re})+\nabla^{2} V_{x}, \\
\nabla \cdot \bar{V}=0 .
\end{gathered}
$$

Причем при записи (7), (8) слагаемых уравнений энергии (4), движения (5) и их критериев подобия принято:

$$
\begin{gathered}
\rho g_{x}-\frac{\partial P}{\partial x}=-g \beta_{\rho} \rho_{0}\left(T-T_{0}\right) \cos a-\frac{\partial}{\partial x}\left(P-P_{0}\right), \\
\mathrm{Fo}=\frac{\kappa \tau}{L^{2}} ; \kappa=\frac{\lambda}{\rho c_{p}} ; \mathrm{Zh}=\frac{v \tau}{L^{2}} ; \mathrm{Zh}=\frac{P e F o}{\operatorname{Re}} ; \\
\mathrm{Eu}=\frac{P}{\rho \bar{V}^{2}} ; \mathrm{Pe}=\operatorname{Re} \mathrm{Pr} ; \mathrm{Gr}=\frac{g \beta_{\rho} \theta_{w} L^{3}}{v^{2}}=G a \beta_{\rho} \theta_{w} .
\end{gathered}
$$

Здесь к - коэффициент температуропроводности; $\tau$ - время процесса; $\lambda$ - коэффициент теплопроводности; $v$ - коэффициент кинематической вязкости; $\rho-$ плотность; $\beta_{\rho}$ - коэффициент объемного расширения.

Так как при исследовании движения флюида, вызываемого разностью плотностей отдельных частиц, измерять скорость затруднительно без специальных средств, полезны следующие обобщения, которые диктуются методом теории подобия гидродинамических и теплодиффузионных явлений. Следуя известному приему сравнения безразмерных дифференциальных уравнений и соответствующих им граничных и начальных условий, описывающих рассматриваемые явления процессов переноса при миграции, вполне определенно в масштабах характерных значений их параметров реализуются безразмерные известные комплексы. Например, при оценках силы тяжести в сранении с инерционными эффектами привлекают не только критерии Фруда ((Fr) как соотношение между силой инерции и внешней силой, в поле которой происходит движение), Ричардсона ((Ri) как мера отношения потенциальной энергии элементов смеси, погружённой в окружающую жидкость, к соответствующей кинетической энергии), но и важные их комбинации с критериями: Галилея ((Ga) как соотношение между силами гравитации и силами вязкости в среде), Архимеда ((Ar) как соотношение между архимедовой силой, обусловленной различием плотностей в отдельных областях рассматриваемой системы, и вязкими силами в основном потоке), Грасгофа ((Gr) как соотношение между подъемной силой, обусловленной разностью плотностей жидкости в отдельных точках потока, и силами молекулярной вязкости) в виде $\mathrm{Ga}=\mathrm{FrRe}^{2}=g L^{3} / v^{2}, \operatorname{Ar}=\mathrm{Ga}\left(\rho-\rho_{0}\right) / \rho$, $\mathrm{Ri}=\mathrm{Ar} / \mathrm{Re}^{2}$. Эти данные ценны для физических заключений о режимах течения, обусловленного выталкивающей силой $\left(\boldsymbol{F}_{\boldsymbol{B}}\right)$. Так, при $\mathrm{Ri}<<1$ принимается, что при движении смеси выталкивающая сила пренебрежима мала, а при Ri>1 превалирует в том значении, что конвективные механизмы мало эффективны при перемещении среды, стратифицированной по плотности.

Более того, учет деталей изменений структуры флюида (при течении гетерогенной среды) требует анализа дополнительных эффектов, определяющих межфазные процессы на границах изменений сруктуры, с помощью таких параметров, как критерий Вебеpa (We $=\rho L_{0} V_{0}^{2} / \sigma-$ отнощение инерции жидкости к поверхностному натяжению), капиллярности $\left(\mathrm{Cp}=\eta V_{0} / \sigma-\right.$ соотношение между вязким трением $(\eta)$ и поверхностным натяжением $(\sigma))$ или их комбинаций типа $\mathrm{We}=\mathrm{CpRe}$. Вместе с этим учет особенностей взаимодействия между внешними силами и силами поверхностного натяжения требует введения критерия Этвеша $\left(\left(\mathrm{E} 0=L / L_{c}\right)\right.$, где $L_{c}=\left(\frac{\sigma}{g \Delta \rho}\right)^{0,5}-$ капиллярная длина) и его комбинации для данной среды типа Eo=ArCp/Re. При выраженной неизотермичности и диффузионности процессов новые неизвестные можно определить по связям для задачи с тепломассообменом: $\mathrm{Gr}=\mathrm{Ga} \beta_{\rho} \Delta T, \quad \mathrm{Fo}_{d}=\mathrm{FoLe}-$ диффузионный критерий 
Фурье. Так $\mathrm{Fo}_{d}$ характеризует соотношение между скоростью изменения диффузионных процессов в окружающей среде и скоростью перестройки концентрационного поля внутри рассматриваемой системы, которые зависят от размеров системы и коэффициента диффузии. Критерий Льюиса Le $=D_{\text {mix }} / \kappa$ характеризует взаимосвязь процессов тепломассопереноса в смесях и является мерой интенсивности изменений диффузионного поля относительно поля температур в смеси, $D_{\text {mix }}-$ коэффициент диффузии смеси.

Полезную для практики информацию по оценке

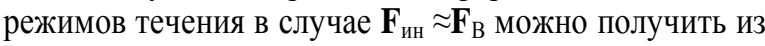
членов уравнения движения (5) $-\frac{\rho \bar{V}^{2}}{2} \approx g L_{x} \Delta \rho$. Последнее, в случае учета переходных тепловых и динамических явлений, при течении флюида в пористой среде приводит к связи $\mathrm{Re} \approx \mathrm{Gr}^{0,5}$.

При оценках сил в рамках допущений II исходим из определяющих уравнений движения в пористой среде при условиях (9):

$$
\operatorname{Re}=\frac{\rho \bar{V} L_{y}}{\mu} \ll 1 ; \rho \frac{d \bar{V}}{d t} \cong 0
$$

и для пористых сред

$$
\operatorname{Re}=\frac{\rho V_{0}}{\mu}\left(\frac{\kappa}{m}\right)^{0,5} \leq 1-10,
$$

где $k$ - проницаемость; $m$ - пористость. Тогда для случая просачивания изотропной ньютоновской среды через пористое твердое тело вполне разумно использовать систему определяющих уравнений (10) в виде $[12,25,26]$ :

$$
\nabla P=\mu \nabla^{2} \bar{V} ; \nabla \cdot \bar{V}=0 .
$$

Заметим, что в рамках допущений II (с учетом (9) и включения силы тяжести) определение скорости флюида по трещине осуществляется в виде (11):

$$
\bar{V}=-\frac{\kappa}{\mu}(\nabla P-\rho \bar{g}) .
$$

Таким образом, детальный анализ процессов при миграции флюида показывает, что на величину выталкивающей силы $\left(\mathbf{F}_{\mathrm{B}}\right)$ может влиять режим течения среды через изменение сил: инерционных $\left(\mathbf{F}_{\text {ин }}\right)$, реологических $\left(\mathbf{F}_{\mathrm{PL}}\right)$, диффузионных $\left(\mathbf{F}_{\mathrm{RC}}\right)$, что допускает формулировку результирующей силы согласно рис. 2 в виде (12):

$$
\bar{F}_{\Sigma}=\bar{F}_{B}+\bar{F}_{G}+\bar{F}_{P L}+\bar{F}_{R C}+\bar{F}_{P R}+\bar{F}_{S D} .
$$

Тогда при оценках выталкивающей силы можно допустить, что $\mathbf{F}_{\mathrm{B}}=\mathbf{F}_{\mathrm{B}}\left(T, c_{i}, P\right)$ и определять ее в виде $\mathbf{F}_{\mathrm{B}}=\mathbf{g}\left(\rho_{0}-\rho(T, P)\right)$, где $\rho_{0}-$ характерная плотность, $\rho(T, P)$ - локальная плотность в области, генерирующей направленную вверх выталкивающую силу $\mathbf{F}_{\mathrm{B}}$, зависящую от изменений термодинамических условий. Также для доминирующих направлений течения в порах имеем полезные для практики оценки (13):

$$
\begin{aligned}
& F_{B x}=-g_{x}\left(\rho_{0}-\rho\right)=|\bar{g}| \cos a\left(\rho_{0}-\rho\right) ; \\
& F_{B y}=-g_{y}\left(\rho_{0}-\rho\right)=|\bar{g}| \sin a\left(\rho_{0}-\rho\right) ;
\end{aligned}
$$

$\rho(T)=\rho_{0}\left[1+\xi\left(T_{0}-T\right)\right] ; \xi-$ коэффициент объемного расширения $\left(\approx 10^{-3}\right)$.

Сила реологических эффектов частии смеси, $\mathbf{F}_{\mathrm{PL}}$. Учет эффектов и процессов, сопровождаемых взаимодействием областей капельной жидкости с нетривиальным характером распределения напряжений и скоростей деформаций, требует уяснения вкладов этих процессов в общий баланс переноса импульса, описываемого уравнением движения в пористой среде и оценок порядка соответствующих сил реологических изменений. Хорошо известно [12, 13, 25, 26], что характерной особенностью течений углеводородных капельных жидкостей со сложной реологией через щелевое пространство породы являются диффузионные эффекты, ответственные за термодинамическую неравновесность всего потока смеси. Аналогичные процессы можно встретить в потоках углеводородов с введением полимерных растворов с целью снижения эффектов молярного переноса импульса при транспортировке природного сырья [27]. Такие кинетические эффекты проявляются в том, что при постоянных внешних условиях происходит затягивание переходов вихревой, тепловой природы, увеличивающих критические значения критериев подобия, отвечающих за неустойчивости, прямые и обратные переходы в потоке (критерии Рейнольдса (Re), Рэлея (Ra)).

В рассматриваемых средах характерное время течения будет превышать гидродинамическое время потока смеси, фиксируемого по пьезопроводности пласта. Для учета этих особенностей в течении реологически сложных сред и определения характера изменений их напряжений наряду с Re, Ra привлекают критерий Дебора (De), характеризующий связь между упругими и вязкими силами. В своем наиболее распространенном виде (рекомендуемом для практических исследований), включающем время структурной релаксации, критерий имеет вид $\mathrm{De}=\lambda V_{0} / L_{y}$, где $\lambda$ отвечает за релаксационные явления в сдвиговых/деформационных процессах и время установления гидродинамического режима течения, которое требуется для снижения начального напряжения в локальной области среды и проявления признаков начала текучести. Кроме того, для описания релаксационных процессов, происходящих при охлаждении углеводородной среды при ее восходящей миграции на поверхность, целесообразно обращение к критериям: Лилли $(\mathrm{Li})-\mathrm{Li}=d \lambda / d t$, где $t$ - характерное время процесса; а также Вейссенберга (Wi), характеризующего особенности изменений вязкоупругого течения соотношением между временем релаксации $(\lambda)$ и скоростью деформаций $(\dot{\varepsilon})$ вида $\mathrm{Wi}=\lambda \varepsilon^{\prime}$.

Отдельные исследования фильтрационных движений тяжелых нефтей $[12,13,18,25,26]$ показывают, что на длинах порядка $L_{y}=10^{-2}$ м скорости $V_{0}$ могут достигать значений $40 \mathrm{~cm} / \mathrm{c}$ и время релаксации определяется диапазоном $\tau=10^{-2}-10^{-1}$, с. Это дает значения $\operatorname{De} \approx O\left(10^{\circ}\right)$ и позволяет утверждать о необходимости учета в результирующей силе реологических эффектов. 
Исходя из сказанного, представляется целесообразным остановиться на отдельных формулировках указанных критериев, по значениям которых можно оценить степень влияния механизмов гидродинамики и тепломассопереноса на динамику процесса течения флюида в пористой среде в условиях, отличных от уравнения (11) (с пренебрежимо малым влиянием инерционных сил $(\operatorname{Re} \leq 1-10)$, течение в изотропной изотермической ньютоновской химически инертной однородной вязкой жидкости в пористой среде).

Прежде всего, следует учитывать, что углеводородные капельные жидкости (тяжелые нефти), не подчиняющиеся закону Дарси и отличающиеся анизотропным характером изменений поверхностных сил $(\operatorname{Re}>10)$, приводят к нелинейной связи уравнения движения, которая усиливается при увеличении проницаемости $(k)$ или уменьшении вязкости. Такие течения анализировались, например, в $[28,29]$, в условиях, когда (11) перестает выполняться, что приводит к учету особенностей влияния пористых сред по модели вида (14):

$$
\bar{V}+\frac{\rho}{\mu} \kappa^{\prime} V^{2}=-\frac{\kappa}{\mu} \nabla P, \kappa=\frac{L_{y}^{2} m^{3}}{150(1-m)} ; \kappa^{\prime}=\frac{1,75 L_{y}}{150(1-m)} .
$$

Здесь $L_{y}-$ характерный поперечный размер пор; $\kappa^{\prime}$ - инерционный коэффициент.

Более того, заметим, что анизотропия и действие реологических эффектов могут усиливаться при интенсификации тепловых процессов $[15,30]$. В этом случае система определяющих уравнений (неразрывности (15), движения (16) и энергии (17)), описывающих гидродинамику и теплообмен в неизотермической углеводородной капельной жидкости, усложняется и принимает вид [31]:

$$
\begin{gathered}
\nabla \cdot \bar{V}=0 ; \\
\bar{V}=-\frac{\kappa}{\mu}(\nabla P-\rho \bar{g}) ; \\
\hat{\sigma} \frac{d T}{d t}=\chi \nabla^{2} T .
\end{gathered}
$$

Здесь $\hat{\sigma}=\frac{m\left(\rho c_{p}\right)_{f}+(1-m)\left(\rho c_{p}\right)_{s}}{\left(\rho c_{p}\right)_{f}}-$ отношение теплоемкости насыщенной среды к теплоемкости жидкости «fл); индекс «S» относится к твердому телу; $\rho=\rho_{0}\left[1-\beta_{\rho}\left(T-T_{0}\right)\right] ; \chi-$ коэффициент температуропроводности жидкости. Кроме того, в данных условиях анизотропию пористых сред (при наличии переходов от свободной к вынужденной конвекции) можно оценить по критериям теплового подобия (Рэлея, $\mathrm{Ra},(18))$ $[28,29,31]$ :

$$
\begin{aligned}
& \left(R a_{\text {horiz }}^{\prime}\right)_{\mathrm{kp}}=\pi^{2}\left[1+\left(\frac{\lambda_{\text {vert }} m_{\text {vert }}}{\lambda_{\text {horiz }} m_{\text {horiz }}}\right)^{0,5}\right]^{2} ; \\
& \left(R a_{\text {vert }}^{\prime}\right)_{\mathrm{kp}}=\pi^{2}\left[1+\left(\frac{\lambda_{\text {horiz }} m_{\text {horiz }}}{\lambda_{\text {vert }} m_{\text {vert }}}\right)^{0,5}\right]^{2} .
\end{aligned}
$$

Здесь $\lambda, m$ - соответственно, коэффициенты теплопроводности, проницаемости в горизонтальном («horiz») и вертикальном («vert») направлениях.

Детальное представление о порядке критических значений критерия $\mathrm{Ra}\left(\mathrm{Ra}=\mathrm{GrPr}\right.$ или $\mathrm{Ra}=\mathrm{RiPrRe}^{2}$, где $\mathrm{Ri}=\mathrm{Gr} / \mathrm{Re}^{2}$ ) в полномасштабных тепло- и гидродинамических конфигурациях, характерных для процессов в глубинных подземных коллекторах (и пористых средах) при переходах тепловой природы, можно получить по следующим заключениям [13, 24, 32]:

- в пространственных протяженных и неизотермических вязких системах достигается критическое значение Рэлея $\mathrm{Ra}_{\mathrm{cr}}=8 \cdot 10^{5}$;

- в скважинных условиях и наличии конвекции имеет место $\mathrm{Ra}_{\mathrm{cr}}=68-216$, причем это критическое значение можно предсказать по (19) с учетом зависимости от отношения теплопроводностей заполняющей скважину жидкости и окружающего ее массива $\lambda_{f} / \lambda_{m}[32]$ :

$$
\begin{gathered}
\mathrm{Ra}_{c r i t}=\frac{96}{5\left(1+7 \lambda_{f} / \lambda_{m}\right)} \times \\
\times\left[\begin{array}{l}
3\left(33+103 \lambda_{f} / \lambda_{m}\right)- \\
-\sqrt{\left.3\left(2567+1479 \lambda_{f} / \lambda_{m}+26927 \lambda_{f} / \lambda_{m}\right)\right)^{2}}
\end{array}\right] .
\end{gathered}
$$

Таким образом, высказанные замечания о положениях и особенностях учета реологии, формулировок критериев Ra c целью установления областей неустойчивости, а также допущений к физической модели рассматриваемого процесса течения и тепломассопереноса в вязком углеводородном флюиде в пористой среде в известном смысле могут служить обоснованием необходимости учета/пренебрежения силами, ответственными за сдвиговые и деформационные эффекты при восходящей миграции подземного флюида.

Сила реакции взаимодействуюших твердых недеформируемых частии, $\mathbf{F}_{\mathrm{RC}}$. Согласно [17, 33-36], процессы переноса, обусловленные этой силой, способны оказывать заметное влияние на локальную структуру течения гомогенной или гетерогенной углеводородной среды в силу выраженных термодинамических и структурных изменений в составе смеси в отдельных зонах течения и общей неравновесности фильтрационного потока. Поэтому для гомогенной смеси изменение этой силы следует учитывать через проявление диффузионных эффектов, определяемых величиной диффузионного потока $i$-й компоненты $\left(j_{i}\right)$ в поперечном направлении области течения, включающем механизмы концентрационной, термо- и бародиффузии, в виде (20) [10, 23]:

$$
\begin{gathered}
j_{i}=\rho \sum_{j=1}^{n} \frac{m_{i} m_{j}}{m^{2}} \times \\
\times D_{i j}\left[\frac{\partial x_{i}}{\partial r}+\left(x_{j}-\frac{x_{j} m_{j}}{m}\right) \frac{\partial \ln p}{\partial r}-\frac{m}{m_{i} x_{i} \rho} D_{i}^{T} \frac{\partial \ln T}{\partial r}\right], \\
i=\overline{1, n} .
\end{gathered}
$$


Здесь $\rho$ - плотность смеси; $x_{i}$ - осредненная мольная доля $i$-й компоненты; $m, m_{i}-$ молекулярные веса смеси и $i$-й компоненты соответственно; $D_{i j}-$ многокомпонентные коэффициенты диффузии; $D_{i}^{T}-$ коэффициент термодиффузии; $p, T$ - осредненные параметры давления и температуры смеси соответственно.

Заметим, что в большинстве исследований подобными эффектами пренебрегают, считая их слабыми. Однако этот вопрос требует более полного анализа для конкретной конфигурации [33]. Подобного мнения, как о силах с эффектами второго порядка в отношении влияния на изменение динамики течения смеси (капельных сред с твердыми частицами) в пористых телах, придерживаются о таких силах, как $\mathbf{F}_{\mathbf{M}}$ - дополнительная вращательная подъемная сила (Магнуса), $\quad \vec{F}_{M}=\frac{\pi}{8} d_{f}^{2} \rho_{f} C_{l M}\left|\vec{u}_{f}-\vec{u}_{p}\right| \frac{\left|\vec{\Omega}\left(\vec{u}_{f}-\vec{u}_{p}\right)\right|}{|\vec{\Omega}|}$, $\vec{\Omega}=\nabla \vec{u}_{f} / 2-\vec{\omega}_{p} ; \mathbf{F}_{\mathbf{S}}-$ дополнительная подъемная сила (Саффмена), $\vec{F}_{S}=C_{l S} \frac{\rho_{f} \pi}{8} d_{p}^{3}\left[\left(\vec{u}_{f}-\vec{u}_{p}\right) \vec{\omega}_{f}\right], \vec{\omega}_{f}=\nabla \times \vec{u}_{f}$, которые выражены в гетерогенных газожидкостных (величины с индексом «f») с твердыми частицами (величины с индексом «р») смесях при вязкостноинерционно-гравитационных режимах на протяженных участках областей течения и тепломассообмена.

Подъемная сила, $\mathbf{F}_{\mathrm{L}}$. Необходимые величины для оценки значений подъёмной силы, создаваемой вращением частиц смеси в реальной жидкости по связи $F_{L}=\frac{1}{2} \rho V^{2} S C_{l}$

Здесь $\rho$ - локальная плотность жидкости; $V$ - скорость частиц флюида относительно среды; $S$ - площадь поверхности частиц флюида; $C_{l}$ - коэффициент подъемной силы. Этот коэффициент может быть определён из экспериментальных данных с учетом сведений о режиме течения (число Рейнольдса, $R e$ ) и коэффициенте вращения (число Россби, Ro $=V_{z 0} / V_{0}$ ). Так, например, для коэффициентов вращения (Ro) от 0,5 до 4,5 коэффициент подъёмной силы $\left(C_{l}\right)$ находится в диапазоне от 0,2 до 0,6 . Заметим, что возникновение эффектов от действия этой силы обусловлено совокупным влиянием на частицы флюида массовых, инерционных и вязких сил, формирующих локальные области переменного по пространству поля давления $[15,30,34,37]$.

Сила сопротивления тяжелых (твердых) частии при движении в жидкости с их характерной локальной скоростью, $U_{P}-\mathbf{F}_{D}$. Ее формулируют в виде $\mathbf{F}_{D}=C_{D}\left(\pi d^{2} / 4\right)\left[\mathbf{U}-\mathbf{U}_{L}\right]\left(U-U_{L}\right) / 2$. Причиной возникновения является разность скорости несущей среды (смесь) и частицы (жидкой/твердой). Причем при $U>U_{L}$ происходит ускорение дисперсной фазы, а при $U<U_{L}$, наоборот, ее торможение. Здесь $C_{D}$ - коэффициент сопротивления частицы с общей связью $C_{D}=f\left(\operatorname{Re}_{L}\right)$, которая в отдельных условиях (требующих эмпирических и других сведений о деталях процесса) сводится, например, $\mathrm{K}$ виду - при $\mathrm{Re}_{L}>130$ $C_{D}=C_{D \text { stokes }}\left(1+0,15 \operatorname{Re}_{L}^{0,687}\right)$. При малых числах Рейнольдса дисперсной фазы $\left(\operatorname{Re}_{L}=\rho\left[\mathbf{U}-\mathbf{U}_{L}\right] d / \mu\right)$ имеем
$C_{D s t o k e s}=24 / \operatorname{Re}_{L}$. Следует учитывать, что в общем случае коэффициент сопротивления - сложная функция многих параметров, например, $C_{D}=C_{D}\left(\operatorname{Re}_{L}, \mathrm{Kn}, M\right.$, $d / 2 R, \Phi, d / h$, теплофизических свойств, состава смеси, неизотермичности и т. д.).

Отмеченное выше позволяет утверждать, что доминирующими силами в рамках физической модели миграции частиц флюида в поровой среде могут выступать следующие.

Полная сила сопротивления частии флюида восходямему потоку со среднемассовой скоростью, $U_{M}$, $\boldsymbol{F}_{\boldsymbol{S D}}$. Выражение силы следует из уравнения движения в проекции на ось Ох и для случая течения вязкой ньютоновской среды имеет вид:

$$
F_{S D}=-\left.\tau_{x y}\right|_{y=L_{y} / 2} \pi L_{x} L_{y},
$$

где компонента тензора напряжений $\left(\tau_{x y}\right)$ имеет вид $\tau_{x y}=\left.\mu\left(\frac{\partial V_{x}}{\partial y}+\frac{\partial V_{y}}{\partial x}\right)\right|_{y=L_{y} / 2}$. Для простоты выкладок, считая режим автомодельным $\left(V_{y} \approx 0\right)$ и учитывая, что течение флюида можно описать уравнением движения вида $\frac{d p}{d x}=\frac{d \tau_{x y}}{d y}$, следует оценка типа (21)

$$
\tau_{x y} \simeq O\left(L_{y}\left(\frac{P_{0}-P_{L}}{L_{x}}\right)\right) .
$$

Сила градиента давления, $\mathbf{F}_{\mathrm{PR}}$. В процессах миграции для оценки порядка величины этой силы следует привлекать определяющие динамические уравнения Навье-Стокса [13, 18-20, 38] вида (22):

$$
\begin{gathered}
\nabla \cdot \bar{V}=0 ; \\
\rho \frac{d \bar{V}}{d t}=-\nabla P+\mu \nabla^{2} \bar{V} .
\end{gathered}
$$

Причем, как отмечалось выше, для пористой среды имеем закон Дарси, который при использовании связей: $\operatorname{Re}=\frac{\rho \bar{V}}{\mu}\left(\frac{\kappa}{m}\right)^{0,5} \leq 1-10, \bar{V}=\frac{Q}{S}$, а также задании переменных $\rho=\rho_{0}, k=k_{0}, m=m_{0}$ позволяет определить $\bar{V}=\operatorname{Re}\left(\frac{m}{\kappa}\right)^{0,5} \frac{\mu}{\rho}$ и, следовательно, оценить $O(\nabla P)$. Тогда имеем, что $\mathbf{F}_{\mathrm{PR}} \approx O\left(\nabla P \cdot L_{x} L_{y}\right)$.

Таким образом, по представленным соотношениям можно оценить результирующую силу $\bar{F}_{\Sigma}$, порядок которой может служить заключением о возможности формирования в пористой среде эффектов, в той или иной степени генерирующих изменения поверхностных и внешних сил, вызывающих восходящую миграцию частиц гомогенного и гетерогенного углеводородного флюида из подземных глубин. В частности, наши оценки по представленной технологической схеме с системными параметрами, характерными для пористой среды в рассматриваемой гидродинамической и тепловой конфигурации, необходимыми для расчета указанных сил, приводят к заключению о доминирующих эффектах и силах при миграции флюида в глубинных горизонтах 
Таблица 1. Определяющие параметры результирующей силы восходящеей миграции флюида

Table 1. Defining parameters of the resulting fluid upward migration force

\begin{tabular}{|c|c|c|c|c|c|c|}
\hline \multicolumn{3}{|c|}{$\begin{array}{c}\text { Переменные области пространства } \\
\text { Space region variables }\end{array}$} & \multicolumn{3}{|c|}{$\begin{array}{c}\text { Характерные параметры углеводородной } \\
\text { капельной среды } \\
\text { Characteristic parameters } \\
\text { of hydrocarbon drip medium }\end{array}$} & $\begin{array}{l}\text { Определяющие критерии гидро- } \\
\text { динамического, теплового и } \\
\text { диффузионного подобия } \\
\text { Defining criteria of hydrodynamic, } \\
\text { thermal and diffusion similarity }\end{array}$ \\
\hline \multirow[b]{3}{*}{$\begin{array}{c}\text { продольное } \\
\text { направление } \\
\text { longitudinal direction } \\
L_{x},(\mathrm{~m})\end{array}$} & \multirow{3}{*}{\multicolumn{2}{|c|}{$10^{3}$}} & \multirow[b]{3}{*}{$\begin{array}{c}\text { Вязкость, } \mu,(\kappa г /(\mathrm{M} \cdot \mathrm{c})) \\
\text { при температуре }(T) \\
\text { и давлении }(P) \\
\text { Viscosity, } \mu,(\mathrm{kg} /(\mathrm{m} \cdot \mathrm{s})) \\
\text { at temperature }(T) \\
\text { and pressure }(P)\end{array}$} & $\begin{array}{c}T=50^{\circ} \mathrm{C} \\
P=15 \mathrm{MPa}\end{array}$ & $2083 \cdot 10^{-7}$ & \multirow[b]{3}{*}{ 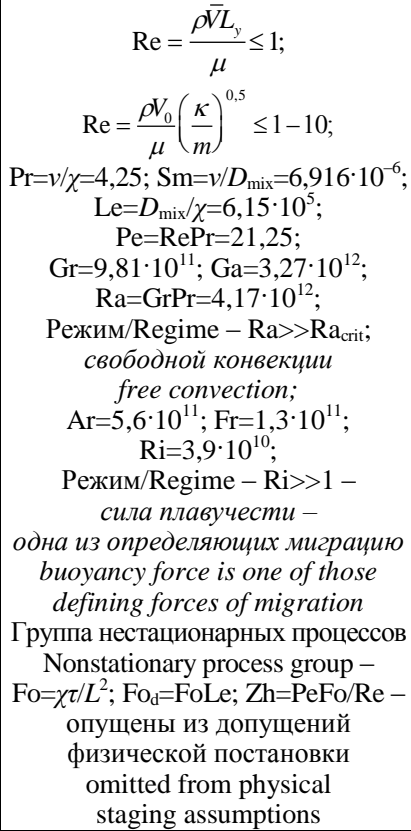 } \\
\hline & & & & $T=25^{\circ} \mathrm{C}$ & & \\
\hline & & & & $P=0,5 \mathrm{MPa}$ & $2172 \cdot 10^{-7}$ & \\
\hline \multirow{3}{*}{$\begin{array}{c}\text { радиальное } \\
\text { направление, } \\
\text { radial direction, } \\
L_{y},(\mathrm{~m})\end{array}$} & \multirow{3}{*}{\multicolumn{2}{|c|}{$(1-2) \cdot 10^{1}$}} & $\begin{array}{c}\text { Плотность } \\
\text { Density, } \rho,\left(\mathrm{kg} / \mathrm{m}^{3}\right) \\
\end{array}$ & \multicolumn{2}{|c|}{626} & \multirow{3}{*}{$\begin{array}{c}\text { Реологические эффекты } \\
\text { Rheological effects - } \\
\text { De }=\tau V_{0} / L_{0} \\
\text { опущен из допущений } \\
\text { физической постановки } \\
\text { omitted from physical } \\
\text { assumptions }\end{array}$} \\
\hline & & & $\rho(T)=\rho_{0}\left[1+\xi\left(T_{0}-T\right)\right]$ & $\begin{array}{r}\xi=10^{-3},\left({ }^{\circ} \mathrm{C}\right) \\
\mathrm{kg} / \mathrm{m}^{3} ; T_{0} \\
\text { (на глубин } \\
T=90^{\circ} \mathrm{C}(\text { нав } \\
\Delta T=\left(T_{0}-T\right.\end{array}$ & $\begin{array}{l}\rho_{0}=626 \\
00^{\circ} \mathrm{C} \\
t \text { depth); } \\
\text { xy/above); } \\
210^{\circ} \mathrm{C}\end{array}$ & \\
\hline & & & & & & \\
\hline \multirow[b]{2}{*}{$\begin{array}{c}\text { азимутальное } \\
\text { направление } \\
\text { transversal direction, } \\
L_{z},(\mathrm{~m})\end{array}$} & \multirow{2}{*}{\multicolumn{2}{|c|}{$10^{-2}$}} & \multirow[b]{2}{*}{$\begin{array}{c}\text { Кинематическая вязкость } \\
\text { Кinematic viscosity, } \\
v=\mu / \rho,\left(\mathrm{m}^{2} / \mathrm{c}\right) \\
\text { при температуре }(T) \\
\text { и давлении }(P) \\
\text { at temperature }(T) \\
\text { and pressure }(P)\end{array}$} & \multicolumn{2}{|c|}{$3,32 \cdot 10^{-7}$} & \\
\hline & & & & \multicolumn{2}{|c|}{$3,47 \cdot 10^{-7}$} & \\
\hline $\begin{array}{c}\text { характерный объем } \\
\text { characteristic volume, } \\
v, \mathrm{~m}^{3}\end{array}$ & \multicolumn{2}{|c|}{$O\left(10^{-2}\right)$} & $\begin{array}{l}\text { Теплоемкость } \\
\text { Heat capacity, } \\
c_{p},\left(\mathrm{~J} /\left(\mathrm{kg} \cdot{ }^{\circ} \mathrm{C}\right)\right) \\
\end{array}$ & \multicolumn{2}{|c|}{2314} & \\
\hline \multirow{4}{*}{$\begin{array}{c}\text { Проницаемость } \\
\text { Permeability, } \\
k,(\text { дарси/darci) } \\
1 \text { darci }= \\
=0,987 \cdot 10^{-12} \mathrm{~m}^{2} \equiv \\
\equiv \mathrm{m \kappa m}^{2}\end{array}$} & $\begin{array}{l}\text { Изотропия } \\
\text { Isotropy }\end{array}$ & $\begin{array}{l}\text { Анизотропия } \\
\text { Anisotropy }\end{array}$ & \multirow{4}{*}{$\begin{array}{l}\text { Температуропровод- } \\
\text { ность } \\
\text { Thermal diffusivity, } \\
\chi=\lambda /\left(\rho \cdot c_{p}\right),\left(\mathrm{m}^{2} / \mathrm{s}\right)\end{array}$} & \multirow{4}{*}{\multicolumn{2}{|c|}{$7,8 \cdot 10^{-8}$}} & \\
\hline & $k=1,5 \cdot 10^{-4}$ & $k_{x}=1,5 \cdot 10^{-4}$ & & & & \\
\hline & $k=4,8 \cdot 10^{-2}$ & $k_{y}=4,8 \cdot 10^{-2}$ & & & & \\
\hline & $k=\bar{A}$ & $\frac{\mu Q L}{\left.P_{1}-P_{2}\right)}$ & & & & \\
\hline \multirow{2}{*}{$\begin{array}{l}\text { Пористость } \\
\text { Porosity, } \\
m,(\%)\end{array}$} & $\begin{array}{r}11,2 \\
\text { (песчани }\end{array}$ & $\begin{array}{l}-16,6 \\
\text { /sandstone) }\end{array}$ & \multirow{2}{*}{$\begin{array}{c}\text { Теплопроводность } \\
\text { Heat conductivity, } \\
\lambda, \mathrm{W} /\left(\mathrm{m}^{\circ}{ }^{0} \mathrm{c}\right)\end{array}$} & \multirow{2}{*}{\multicolumn{2}{|c|}{0,1134}} & \\
\hline & $\begin{array}{r}40 \text { (пе } \\
\text { зернистост }\end{array}$ & $\begin{array}{l}\text { oK/sand, } \\
\text { b/grit 60-70) }\end{array}$ & & & & \\
\hline \multirow[t]{4}{*}{ Угол/angle, a } & \multicolumn{2}{|c|}{$30-45^{\circ}$} & $\begin{array}{c}\text { Диффузия } \\
\text { Diffusion, } D_{\text {mix }},\left(\mathrm{m}^{2} / \mathrm{s}\right) \\
\end{array}$ & \multicolumn{2}{|c|}{0,048} & \\
\hline & & & $\begin{array}{l}\text { Термодинамические } \\
\text { условия на глубине } \\
\text { Thermodynamic } \\
\text { conditions at depth } \\
\end{array}$ & \multicolumn{2}{|c|}{$\begin{array}{c}\mathrm{P}_{0}=30 \mathrm{MPa} ; \mathrm{T}_{0}=300^{\circ} \mathrm{C} \\
\rho_{0}=221-620 \mathrm{~kg} / \mathrm{m}^{3}\end{array}$} & \\
\hline & & & \multirow{2}{*}{$\begin{array}{c}\text { Характерная среднемас- } \\
\text { совая скорость смеси } \\
\text { Characteristic average } \\
\text { mass velocity } \\
\text { of the mixture, } \mathrm{UM}, \mathrm{m} / \mathrm{s}\end{array}$} & $\begin{array}{l}\operatorname{Re}=\frac{\rho V_{0}}{\mu}\left(\frac{\kappa}{m}\right) \\
\text { gives at } \operatorname{Re}=5 \\
\rho=626, m=0,\end{array}$ & $\begin{array}{l}\text { дает при } \\
\iota=2,7 \cdot 10^{-7}, \\
; \kappa=0,025\end{array}$ & \\
\hline & & & & $\bar{V}=\operatorname{Re} \frac{\mu}{\rho}\left(\frac{m}{\kappa}\right)$ & $=3,9 \cdot 10^{-9}$ & \\
\hline
\end{tabular}


Таблица 2. Сведения оиенок сил

Table 2. Force estimates information

\begin{tabular}{|c|c|c|c|c|c|c|c|c|}
\hline \multicolumn{2}{|c|}{$\begin{array}{l}\text { Тяжести } \\
\text { Gravity } \\
\mathbf{F}_{\mathrm{G}}, \mathbf{N}\end{array}$} & $\begin{array}{l}\text { Подъемная } \\
\text { Lifting } \\
\mathbf{F}_{\mathrm{L}}, \mathbf{N}\end{array}$ & \multicolumn{2}{|c|}{$\begin{array}{c}\text { Плавучести } \\
\text { Вuоуаnсу } \\
\mathbf{F}_{\mathrm{B}}, \mathbf{N}\end{array}$} & $\begin{array}{c}\text { Сопротивления } \\
\text { Resistance } \\
\text { F }_{\mathrm{SD}}, \mathrm{N}\end{array}$ & $\begin{array}{c}\text { Перепада } \\
\text { давления } \\
\text { Pressure } \\
\text { difference } \\
\text { F }_{\mathrm{PR}}, \mathbf{N}\end{array}$ & $\begin{array}{c}\text { Реологиче- } \\
\text { ских } \\
\text { эффектов } \\
\text { Rheological } \\
\text { effects } \\
\text { FेL }_{\text {PL }}\end{array}$ & \begin{tabular}{|c} 
Реакции взаимодей- \\
ствующих твердых \\
недеформируемых \\
частиц \\
Reactions of interacting \\
solid non-deformable \\
particles \\
F $_{\mathrm{RC}}, \mathrm{N}$ \\
\end{tabular} \\
\hline \multicolumn{2}{|c|}{$\begin{array}{c}\bar{F}_{G}=-\bar{g} \\
F_{x}=g_{x}= \\
=-|g| \cos a \\
F_{y}=g_{y}= \\
=-|g| \sin a\end{array}$} & $F_{L}=\frac{1}{2} \rho V^{2} S C_{l}$ & \multicolumn{2}{|c|}{$\begin{array}{c}\bar{F}_{B}=\bar{g}\left(\rho_{0}-\rho\left(T, P, c_{i}\right)\right) ; \\
F_{B x}=\left|\bar{F}_{B}\right| \cos a ; \\
F_{B y}=\left|\bar{F}_{B}\right| \sin a\end{array}$} & $\begin{array}{c}F_{S D}=-\left.\tau_{x y}\right|_{y=L_{y} / 2} \pi L_{x} L_{y} \\
\tau_{x y}=\left.\mu\left(\frac{\partial V_{x}}{\partial y}+\frac{\partial V_{y}}{\partial x}\right)\right|_{y=L_{y} / 2}\end{array}$ & $F_{P R}=\left(\nabla P \cdot L_{x}\right) S$ & $F_{P L}=O(\mathrm{De})$ & \begin{tabular}{|c} 
Диффузионные про- \\
цессы, описываемые в \\
рамках неравновесной \\
термодинамики \\
Diffusion processes de- \\
scribed within non- \\
equilibrium thermody- \\
namics \\
\end{tabular} \\
\hline \multicolumn{2}{|c|}{$\angle a=30-45^{\circ}$} & $\begin{array}{c}\rho=757,46 ; \\
C_{\mathrm{l}}=1,005\end{array}$ & \multicolumn{2}{|c|}{$\begin{array}{c}\angle a=30-45^{\circ} ; \\
\rho=757,46 ; \rho_{0}=626\end{array}$} & $\pi S=L_{x} \cdot L_{y}=3,14 \cdot 10^{4}$ & $\nabla P=-\mu \frac{\bar{V}}{\kappa}$ & $\begin{array}{c}\mathrm{Re}=5, \\
\mathrm{Ra}=4,17 \cdot 10^{12}, \\
\mathrm{De}=\tau V_{0} / L_{y}= \\
=O\left(10^{-11}\right)\end{array}$ & \\
\hline$F_{x}$ & $F_{y}$ & & $g_{x}$ & $g_{y}$ & $\mu=2,7 \cdot 10^{-7}$, & $\bar{V}=\operatorname{Re} \frac{\mu}{\rho}\left(\frac{m}{\kappa}\right)^{0,5}$ & & \\
\hline 8,49 & 4,09 & $V=3,9 \cdot 10^{-9}$ & 8,49 & 4,09 & $\begin{array}{c}V_{x} \simeq O(\bar{V}), V_{y} \simeq 0 \\
V=3,910^{-9}\end{array}$ & $\begin{array}{c}\mu=2,7 \cdot 10^{-7} \\
\kappa=0,025 \\
\operatorname{Re}=5\end{array}$ & & \\
\hline \multirow[t]{4}{*}{6,93} & 6,93 & $F_{L}=6,09 \cdot 10^{-11}$ & 6,93 & 6,93 & $L_{y} \simeq O\left(10^{1}\right)$ & $\begin{array}{c}S=L_{x} \cdot L_{y}=10^{4} ; \\
L_{x}=10^{3}\end{array}$ & & \\
\hline & & & $F_{B x}$ & $F_{B y}$ & $\tau_{x y}=O\left(\mu \frac{V}{L_{y}}\right)$ & $V=3,9 \cdot 10^{-9}$ & & \\
\hline & & & $1.11 \cdot 10^{3}$ & $0,53 \cdot 10^{3}$ & $1,053 \cdot 10^{-16}$ & $\nabla P=0,421 \cdot 10^{-13}$ & & \\
\hline & & & $0,91 \cdot 10^{3}$ & $0,91 \cdot 10^{3}$ & $F_{S D}=3,3 \cdot 10^{-12}$ & $F_{P R}=0,421 \cdot 10^{-6}$ & & \\
\hline \multicolumn{2}{|c|}{$F_{g} \simeq O\left(10^{1}\right)$} & $F_{L} \simeq O\left(10^{-11}\right)$ & \multicolumn{2}{|c|}{$F_{B} \simeq O\left(10^{3}\right)$} & $F_{S D} \simeq O\left(10^{-12}\right)$ & \begin{tabular}{|c|}
$F_{P R} \simeq O\left(10^{-6}\right)-$ \\
без учета вязких \\
сил (подход II) \\
without considera- \\
tion of viscous \\
forces (approach II); \\
$F_{P R} \simeq O\left(10^{-11}\right)-$ \\
с учетом вязких \\
сил (подход I) \\
taking into account \\
viscous forces \\
(approach I)
\end{tabular} & $F_{P L} \simeq O\left(10^{-11}\right)$ & $\begin{array}{c}\text { Эффекты силы опу- } \\
\text { щены по допущениям } \\
\text { физической модели, } \\
\text { но целесообразны при } \\
\text { численном моделиро- } \\
\text { вании } \\
\text { Effects of force are } \\
\text { omitted according to the } \\
\text { assumptions of the } \\
\text { physical model, but are } \\
\text { expedient in numerical } \\
\text { modeling }\end{array}$ \\
\hline
\end{tabular}

Из результатов табл. 1, 2 следует, что выполненные оценки порядков сил, сведений о числах подобия (например, $\mathrm{Pr}, \mathrm{Sm}, \mathrm{Le}, \mathrm{Re}, \mathrm{Pe}, \mathrm{Ra}, \mathrm{Ri}$ и т. д.) позволяют выделить определяющие эффекты в результирующей силе (от изменений $\mathbf{F}_{\mathrm{B}}, \mathbf{F}_{\mathrm{G}}, \mathbf{F}_{\mathrm{PR}}$ ), формирующие процесс миграции. А также использовать эти данные в качестве разумного физического и математического инструментов прогноза миграции особенно в условиях, осложненных: аномальными фазовыми и реологическими мелкомасштабными диффузионными изменениями состава; сопряженным тепломассопереносом между породой и смесью; нестабильностью режимов в локальных областях течений флюида при произвольном тектоническом нарушении литосферы.

Приведем пример возможного образования порядка гидростатической силы при появлении глубинного тектонического разлома с вертикальной миграцией флюида. Известно, что литостатическое давление на глубине $\sim 5$ км соответствует $\sim 10^{8}$ Па. В этом случае при появлении разлома глубиной 3 км (например, от фундамента до «бажена»), длиной 1 км при ширине в 1 см (площадь сечения вертикального канала миграции флюида будет соответствовать $10 \mathrm{~m}^{2}$ ) появляется градиент гидростатического давления, направленного вверх. В рамках данной геометрической модели, без учета эффектов вязкого трения, сила гидростатического давления флюида на глубине 2 км будет соответствовать порядкам $10^{5}-10^{6}$ Н. Что, в свою очередь, может служить подтверждением возможности доминирующего или соразмерного влияния эффектов от силы градиента давления на формирование результирующей силы. Поэтому в определенных конфигурациях, режимах гидродинамики и тепломассопереноса справедливо ожидать миграции в условиях как роста вклада от силы градиента давления, так и экстенсификации процессов от сил плавучести и тяжести, а также присутствия других гидродинамических эффектов от рассматриваемых сил. Все это ставит вопросы для дальнейшего детального анализа процессов в рамках совершенных физических и математических моделей. 


\section{Выводы}

1. Настоящий анализ показывает, что эффекты коррекции процессов в подъемной силе за счет изменений дополнительных ее составляющих $\left(\mathbf{F}_{\mathrm{S}}, \mathbf{F}_{\mathrm{M}}\right)$ в рамках выполненного физического анализа (согласно схеме рис. 2) следует считать пренебрежимо малыми.

2. Из данных табл. 1, 2 видно, что восходящая миграция формируется под определяющим влиянием в результирующей силе выталкивающей силы $\left(\mathbf{F}_{\mathrm{B}}\right)$ особенностей изменения полей термодинамических параметров (состава $\left(c_{i}\right)$, полей температуры $(T)$, давления $(P)$, под действием силы тяжести $\left(\mathbf{F}_{\mathrm{G}}\right)$, фазовых явлений и процессов на границах исследуемого объема текущей углеводородной среды), а также силы градиента давления $\left(\mathbf{F}_{\mathrm{PR}}\right)$, поведение которой осложняется распределением всего спектра внешних и внутренних сил в сопряженной системе.

3. Полученные данные могут быть использованы при построении детальной численной математической модели для многопараметрического анализа рассматриваемых пространственных процессов переноса импульса, тепла и массы в пористой среде при восходящей миграции. Такой анализ может стать предметом будущих исследований.

\section{Тенденции и перспективы исследования}

1. Общий анализ рассмотренных проблем моделирования процессов миграции глубинного флюида подчеркивает физическую и математическую нетривиальность задачи в силу своей многопараметричности, многомерности и многофакторности. В таких условиях на пути построения универсальной модели положения теории подобия, метода анализа размерностей органично выступают инструментом обоснованной формулировки заключений о доминирующих процессах, определяющих/второстепенных эффектах/механизмах, сопровождающих миграцию. Анализ характера распределений определяющих чисел, критериев по-

\section{СПИСОК ЛИТЕРАТУРЫ}

1. Дюнин В.И., Корзун В.И. Гидрогеодинамика нефтегазоносных бассейнов. - М.: Научный мир, 2005. - 524 с.

2. Рахматулин Х.А. Основы газовой динамики взаимопроникающих движений сплошных сред // Прикладная математика и механика. - 1956. - Т. 20. - № 2. - С. 54-69.

3. Мельник И.А. Интенсивности процессов наложенного эпигенеза как индикаторы нефтенасыщенности песчаных коллекторов // Известия ТПУ. Инжиниринг георесурсов. - 2019. Т. 330. - № 6. - С. 90-97.

4. Мельник И.А., Зимина С.В., Смирнова К.Ю. Нефтегазоносность территории Томской области как результат глубинной миграции // Геология, геофизика и разработка нефтяных и газовых месторождений. - 2017. - № 3. - С. 17-22.

5. Тимурзиев А.И. Глубинная «фундаментальная нефть» Западной Сибири - реальное состояние и альтернативы развития // Глубинная нефть (электронное издание). - 2014. - Т. 2. № 12. - C. 1951-1970. URL: http://journal.deepoil.ru/images/stories/ docs/DO-2-12-2014/0_DO_Contents_2-12-2014.pdf (15.12.2020).

6. Абукова Л.А. Нисходящая миграция поземных вод и углеводородов в осадочных нефтегазоносных бассейнах // Геология, добия тепловой, гидродинамической и диффузионных задач, установления их функциональных связей в виде уравнений подобия, полученных с привлечением теории подобия, весьма важен при построении точных и приближенных моделей восходящей миграции, детального и комплексного физико-математического описания течений сопряженного ТМО для гомогенных и гетерогенных флюидов в узких полостях в полях действия внешних сил, а также при разработке корректных и эффективных методов их решений, выдаче рекомендаций в практику приложений.

2. Библиографический анализ показывает, что уровень выполненных работ неоднороден и целесообразен труд по обобщению интенсивных усилий исследователей по комплексному моделированию течений из больших глубин с целью понимания фундаментальных аспектов явлений переноса и механизмов течения УВ в замкнутых объемах со сложным составом и реологией.

3. Интерпретация имеющегося теоретического материала направлена на обобщение и развитие сведений о транспорте УВ смесей сложной реологии и состава из замкнутых областей до современного уровня анализа влияния результирующей силы. Требуется дополнительное изучение влияния неустойчивостей, хаотических изменений в структуре смеси, сопутствующих эффектов, характеризующих производство энтропии, ее кривизны (согласно идеям И. Пригожина и П. Гленсдорфа).

4. Движения, возникающие в одно- и многофазной средах из-за разности плотностей в УВ смеси, также осложнены и генерируются различиями фазовых состояний, ТМП в инертной/химически активной среде, существованием «тонких» скрещивающихся механизмов - дино-, баро-, термодиффузии, диффузионной теплопроводности. Это требует тщательных оценок при рассмотрении конкретной конфигурации течения и ТМП. Поэтому все высказанные замечания могут составить предмет перспективных исследований.

геофизика и разработка нефтяных и газовых месторождений. -2010 . - № 8. - С. 23-31.

7. Геокатализ и эволюция мантийно-коровых магматогенных флюидных систем / В.Н. Шарапов, Г.К. Ионе, М.П. Мазуров, В.П. Мысов, Ю.В. Перепечко. - Новосибирск Академическое издание «Гео», 2007. - $141 \mathrm{c}$.

8. Модельный анализ развития континентальных мантийнокоровых рудообразующих систем / В.Н. Шарапов, А.С., Борисенко М.П. Мазуров, Ю.В. Перепечко, А.Н. Черепанов, Ю.П. Бессонова, Г.Г. Павлова, А.А. Боровиков, Л.М. Житова, В.А. Пономарчук, В.Н. Попов, В.К. Черепанова, К.В. Чудненко. - Новосибирск: Изд-во СО РАН, 2009. - 150 с.

9. Мельник И.А. Определение интенсивности геохимических процессов по материалам геофизических исследований скважин. - Новосибирск: СНИИГГиМС, 2016. - 146 с.

10. Де Гроот С., Мазур П. Неравновесная термодинамика. - М.: Наука, 1970. - 231 c.

11. Нигматулин Р.И. Динамика многофазных сред. Ч. 1. - М.: Наука, 1987. - 464 с.

12. Маскет М. Течения однородных жидкостей в пористой среде. - М.; Ижевск: Институт компьютерных исследований, 2004. $-640 \mathrm{c}$. 
13. Петухов Б.С. Теплообмен и сопротивление при ламинарном течении жидкости в трубах. - М.: Энергия, 1967. - 411 с.

14. Кутателадзе С.С., Стырикович М.А. Гидрогазодинамика газожидкостных систем. - М.: Энергия, 1976. - 296 с.

15. Advances of heat transfer in porous media - a review / Younes Menni, Ali J. Chamkha, Noureddine Kaid, Houari Ameur, Mohammed Bensafi, Djamel Sahel, Giulio Lorenzini // Special Topics \& Reviews in Porous Media: an International Journal. - 2020. V. 11. - Iss. 1. - P. 1-18. DOI: 10.1615/SpecialTopicsRevPorous Media.2020028581

16. Deepak Kumar, Amit Kumar Dhiman. Effects of aiding buoyancy and channel confinement on the flow and heat transfer of dilatant fluids from a square obstacle // Computational Thermal Sciences: an International Journal. - 2018. - V. 10. - Iss. 2. - P. 121-149. DOI: 10.1615/ComputThermalScien. 2017021128.

17. A critical review of engineering approach in the context of memory concept for fluid flow through porous media / Fatema Akter Happy, Arifur Rahman, Syed Imtiaz, M. Enamul Hossain // Journal of Porous Media. - 2020. - V. 23. - Iss. 6. - P. 593-611. DOI: 10.1615/JPorMedia. 2020020571

18. Kharlamov S.N., Malozemov A.V. Investigation of regularities highly viscous liquid flows in interaction with peripheral annular flow of water-oil in complex pipelines // Applied Mechanics and Materials. - 2014. - V. 565. - P. 152-155. DOI: 10.4028/www.scientific.net/AMM.565.152

19. Терехов В.И., Пахомов М.А. Тепломассоперенос и гидродинамика в газокапельных потоках. - Новосибирск: Изд-во НГТУ, 2009. - 284 с.

20. Накоряков В.Е., Покусаев Б.Г., Шрейбер И.Г. Волновая динамика газо- и парожидкостных систем. - М.: Энергоатомиздат, 1990. $-321 \mathrm{c}$.

21. Surface wetting in multiphase pipe-flow / J.R. Bentzon, A. Vural, K.L. Feilberg, J.H. Walther // Multiphase Science and Technology. 2020. - V. 32. - Iss. 2. - P. 137-154. DOI: 10.1615/MultScien Techn. 2020031539

22. Hosokawa Sh., Shakutsui H., Tomiyama A. Turbulence modification of gas-liquid solid dispersed three-phase flow in a vertical pipe // Multiphase Science and Technology. - 2019. - V. 31. Iss. 2. - P. 175-197. DOI: 10.1615/MultScienTechn. 2019030437.

23. Бубенчиков А.М., Харламов С.Н. Математические модели неоднородной анизотропной турбулентности во внутренних течениях. - Томск: Томский государственный университет, 2001. -448 c.

24. Петухов Б.С., Поляков А.Ф. Теплообмен при смешанной турбулентной конвекции. - М.: Наука, 1986. - 192 с.

25. Маскет М. Физические основы технологии добычи нефти. М.; Ижевск: Институт компьютерных исследований, 2004. $608 \mathrm{c}$.

26. Мирзаджанзаде А.Х., Хасанов М.М., Бахтизин Р.Н. Моделирование процессов нефтегазодобычи. Нелинейность, неравновесность, неопределенность. - М.; Ижевск: Институт компьютерных исследований, 2004. - 368 с.

27. Kharlamov S.N., Kudelin N.S., Dedeev P.O. Hydrodynamic, heat and acoustic processes modeling in transport of rheologically complex viscous media technology in pipelines // XVIII International scientific symposium in honour of academician M.A. Usov. IOP Publishing, IOP conf. series: Earth and environmental science - 2014 - V. 21 - № 012040 - P. 1-7. DOI: 10.1088/1755$1315 / 21 / 1 / 012040$

28. Benjan A., Khair K.R. Heat and mass transfer by natural convection in a porous medium // International journal of heat and mass transfer. - 1985. - V. 28. - Iss. 5. - P. 909-918.

29. Hong J.T., Tien C.L., Kaviani M. Non-Darcian effects on vertical plate natural convection in porous media with high porosities // International journal of heat and mass transfer. - 1985. - V. 28. Iss. 12. - P. 2149-2159.

30. Dhananjay Yadav. Numerical examination of the thermal instability in an anisotropic porous medium layer subjected to rotation and variable gravity field // Special Topics \& Reviews in Porous Media An International Journal. - 2020. - V. 11. - Iss. 1. - P. 395-407.

31. Larson R.E., Higdon J.J.L. Microscopic flow near the surface of two-dimensional porous media. P. 2 Transverse flow // Journal of Fluid Mechanics. - 1987. - V. 178. - P. 119-136.

32. Гершуни Г.З., Жуховицкий Е.М. Конвективная устойчивость несжимаемой жидкости. - М.: Наука, 1972. - 392 с.

33. Obembe Abiola David, M. Enamul Hossain, Sidqi A. AbuKhamsin. Variable-order anomalous heat transport mathematical models in disordered and heterogeneous porous media // Second Thermal and Fluids Engineering Conference. - Las Vegas, NV, USA, 2017, 2-5 April. - P. 2225-2239. DOI: 10.1615/ TFEC2017.prm.017616.

34. Chetteti Ram Reddy, Padigepati Naveen, Darbhasayanam Srinivasacharya. Effects of nonlinear convection and cross-diffusion for the flow of darcy-forchheimer model micropolar fluid with convective boundary condition // Computational Thermal Sciences: an International Journal. - 2019. - V. 11. - Iss. 3. - P. 205-218. DOI: 10.1615/ComputThermalScien.2018019453.

35. Two-way coupled fluid - structure interaction of gas-liquid slug flow in a flexible riser: small-scale experiments and simulations / J.J. Vieiro, A. Akhiiartdinov, S. Sævik, C.M. Larsen, O.J. Nydal // Multiphase Science and Technology. - 2019. - V. 31. - Iss. 1. P. 27-43. DOI: 10.1615/MultScienTechn.2019029489.

36. Saman Beyhaghi, Krishna Pillai. Estimation of tortuosity and effective diffusivity tensors using closure formulation in a sintered polymer wick during transport of a nondilute, multicomponent liquid mixture // Special Topics \& Reviews in Porous Media: an International Journal. - 2011. - V. 2. - Iss. 4. - P. 267-282. DOI: $10.1615 /$ SpecialTopicsRevPorousMedia.v2.i4.20.

37. Majid Molki. The swirling motion of dribbling honey // 5th Thermal and Fluids Engineering Conference (TFEC). - New Orleans, LA, USA, 2020, 5-8 April. - P. 321-332. DOI: 10.1615/ TFEC2020.fnc.031576.

38. Kharlamov S.N. Characteristics of flow and heat transfer in a turbulent twisted flow // Heat Transfer Research. - 2002. - V. 33. Iss. 1-2. - P. 64-74.

Поступила 23.12.2021 2.

\section{Информация об авторах}

Мельник И.А., доктор геолого-минералогических наук, профессор, и.о. заведующего кафедрой-руководителя отделения нефтегазового дела на правах кафедры Инженерной школы природных ресурсов Национального исследовательского Томского политехнического университета.

Харламов С.Н., доктор физико-математических наук, профессор, профессор отделения нефтегазового дела Инженерной школы природных ресурсов Национального исследовательского Томского политехнического университета. 
UDC 553.982:551.252:536.24:532.54

\title{
ON THE ISSUE OF HYDRODYNAMIC MODELLING OF UPWARD MIGRATION OF RHEOLOGICALLY COMPLEX INHOMOGENEOUS FLUIDS FROM DEEP UNDERGROUND RESERVOIRS
}

\author{
Igor A. Melnik', \\ melnik@tpu.ru \\ Sergey N. Kharlamov', \\ kharsn@mail.ru \\ 1 National Research Tomsk Polytechnic University, \\ 30, Lenin avenue, Tomsk, 634050, Russia.
}

\begin{abstract}
The relevance of the article is caused by the need to develop new mathematical models, methods, experimental approaches when investigating migration problems of deep hydrocarbon fluids from underground reservoirs of the oil and gas basin.

The aim of the research is to develop a universal physical and mathematical model of a detailed forecast of migration over a limited space of a ground collector of arbitrary geometry; make recommendations to the practice of studying the problems of hydrodynamics and heatand mass transfer in these conditions and to compare the relative values of forces that cause the migration of deep fluids in vertical and lateral directions.

Methods of the research are based on general principles of mechanics of continuous homogeneous and heterogeneous media, hydrodynamics and heat and mass transfer.

Results. The paper introduces the data of analysis of effects accompanying hydro- and gas dynamics, and heat- and mass transfer at flows of homogeneous and heterogeneous rheologically simple and complex viscous mixtures in fields of action of external forces from underground regions with arbitrary geometry of slot and pore space. The authors have carried out the analysis of the correctness of the physical modeling of the problem on underground hydrocarbons migration and establishment of determining mechanisms for their lifting from large depths. It was found that upward migration is formed under the defining influence in the resulting force: floating force $\left(F_{B}\right)$, which takes into account the features of changes in the fields of thermodynamic parameters (composition $\left(c_{i}\right)$, temperature $(T)$ ), pressure $(P)$ ); gravity $\left(\boldsymbol{F}_{G}\right)$ : phase phenomena and processes at the boundaries of the analysed volume of the hydrocarbon medium, as well as pressure gradient force $\left(\boldsymbol{F}_{P R}\right)$, the behavior of which is complicated by the distribution of the entire spectrum of external and internal forces in the conjugated system. The paper introduces the conclusions on details of fluid flow modeling, formulations of defining numbers and criteria within the framework of the theory of similarity of the considered problems, useful for understanding and clarifying the essence of effects, processes, forces, mechanisms accompanying migration. The results can be used to predict migration especially at the conditions complicated by: abnormal phase and rheological small-scale diffusion changes in composition; conjugated heat and mass transfer between rock and mixture; instability of modes in local areas of fluid flow at arbitrary thermodynamic and geometric configurations of underground collector.
\end{abstract}

\section{Key words:}

Fluid, mixtures, migration, modeling, hydrodynamics, heat- and mass transfer, rheology, structure, mass forces.

\section{REFERENCES}

1. Dunin V.I., Korzun V.I. Gidrogeodinamika neftegazonosnykh basseynov [Hydrogeodynamics of oil and gas bearing basin]. Moscow, Naushny mir Publ., 2005. 524 p.

2. Rakhmatulin Kh.A. Osnovy gazovoy dinamiki vzaimopronikayushchikh dvizcheniy sploshnykh sred [Fundamentals of gas dynamics of interpenetrating flows of continuous media]. Applied mathematics and mechanics, 1956, vol. 20, no. 2, pp. 54-69.

3. Melnik I.A. Intensity of processes of superimposed epigenesis as indicators of oil saturation of sand reservoirs. Bulletin of the Tomsk Polytechnic University. Geo Assets Engineering, 2019, vol. 330, no. 6, pp. 90-97. In Rus.

4. Melnik I.A., Zimina S.V., Smirnova K.Yu. Oil and gas potential of the Tomsk region as a result of deep migration. Geology, geophysics and development of oil and gas field, 2017, no. 3, pp. 17-22. In Rus.

5. Timurziev A.I. The deep «fundamental oil» of Western Siberia the real state and alternatives to development. The deep oil (electronic edition), 2014, vol. 2, no. 12, pp. 1951-1970. In Rus. Available at: http://journal.deepoil.ru/images/stories/docs/DO-2-12-2014/ 0_DO_Contents_2-12-2014.pdf (accessed 15 December 2020).

6. Abukova L.A. Downward migration of terrestrial waters and hydrocarbons in sedimentary oil and gas basins. Geology, geophysics and development of oil and gas field, 2010, no. 8, pp. 23-31. In Rus.

7. Scharappov V.N., Ione G.K., Mazurov M.P., Mysov V.P., Perepchenko Yu.V. Geokataliz i evolutsiya mantyino-korovykh magmatogennykh flyuidnykh sistem [Geocatalysis and evolution of mantle-core magmatogenic fluid systems]. Novosibirsk, Geo Akademik Publ., 2007. 141 p.

8. Scharapov V.N., Borisenko F.S., Masurov M.P., Perepechko Yu.V., Cherepanov A.N., Bessonova Yu.P., Pavlova G.G., Borovikov A.A., Zhitova L.M., Ponomarchuk V.A., Popov V.N., Cherepanova V.K., Chudnenko K.V. Modelny analiz rasvitiya kontinentalnykh mantiyno-korovykh rudoobrazuyushchikh sistem [Model analysis of development of continental mantle-crust ore-forming systems]. Novosibirsk, SB RAN Publ., 2009. 150 p.

9. Melnik I.A. Opredelenie intensivnosti geokhimicheskikh protseccov po materialam geofizicheskikh issledovaniy skvazchin [Determination of geochemical processes intensity based on materials of well geophysical surveys]. Novosibirsk, SNIIGGiMS Publ., 2016. $146 \mathrm{p}$.

10. De Groot S., Mazur P. Neravnovesnaya termodinamika [Nonequilibrium thermodynamics]. Moscow, Nauka Publ., 1970. 231 p.

11. Nigmatullin R.I. Dinamika mnogofaznykh sred [Dynamics of multi-phased media]. P. 1. Moscow, Nauka Publ., 1987. 464 p.

12. Musket M. Techenie odnorodnykh zchidkostey $v$ piristoy srede [Flows of homogeneous liquids in porous medium]. Moscow, Izhevsk, Institute for Computer Research Publ., 2004. 640 p.

13. Petukhov B.S. Teploobmen i soprotivlenie pri laminarnom techenii zchidkosti $v$ trubakh [Heat exchange and resistance at laminar flow of liquid in pipes]. Moscow, Energiya Publ., 1967. 411 p.

14. Kutataladze S.S., Styrikovich M.A. Gidrogazodinamika gazozhidkostnykh sistem [Hydrogasodynamics of gas-liquid systems]. Moscow, Energiya Publ., 1976. 296 p.

15. Younes Menni, Ali J. Chamkha, Noureddine Kaid, Houari Ameur, Mohammed Bensafi, Djamel Sahel, Giulio Lorenzini. Advances of 
heat transfer in porous media - a review. Special Topics \& Reviews in Porous Media: an International Journal, 2020, vol. 11, Iss. 1, pp. 1-18. DOI: 10.1615 / SpecialTopicsRevPorousMedia.2020028581

16. Deepak Kumar, Amit Kumar Dhiman. Effects of aiding buoyancy and channel confinement on the flow and heat transfer of dilanant fluids from a square obstacle. Computational Thermal Sciences: an International Journal, 2018, vol. 10, Iss. 2, pp. 121-149. DOI: 10.1615/ComputThermalScien.2017021128

17. Fatema Akter Happy, Arifur Rahman, Syed Imtiaz, M. Enamul Hossain. A critical review of engineering approach in the context of memory concept for fluid flow through porous media. Journal of Porous Media, 2020, vol. 23, Iss. 6, pp. 593-611. DOI: 10.1615/JPorMedia.2020020571

18. Kharlamov S.N., Malozemov A.V. Investigation of regularities highly viscous liquid flows in interaction with peripheral annular flow of water-oil in complex pipelines. Applied Mechanics and Materials, 2014, vol. 565, pp. 152-155. DOI: 10.4028/www. scientific.net/AMM.565.152 (ISSN: 1022 - 6680).

19. Terekhov V.I., Pakhomov M.A. Teplomassoperenos i gidrodinamika $v$ gazokapelnom potoke [Heat and mass transfer and hydrodynamics in gas-droplet flows]. Novosibirsk, NGTU Publ., 2009. $284 \mathrm{p}$.

20. Nakoryakov V.E., Pokusaev B.G., Schreiber I.G. Volnovaya dinamika gazo- i parozhidkostnykh sistem [Wave dynamics of gas and steam-liquid systems]. Moscow, Energoatomizdat Publ., 1990. $321 \mathrm{p}$.

21. Bentzon J.R., Vural A., Feilberg K.L., Walther J.H. Surface wetting in multiphase pipe-flow. Multiphase Science and Technology, 2020, vol. 32, Iss. 2, pp. 137-154. DOI: 10.1615/MultScienTechn. 2020031539

22. Hosokawa Sh., Shakutsui H., Tomiyama A. Turbulence modification of gas-liquid solid dispersed three-phase flow in a vertical pipe. Multiphase Science and Technology, 2019, vol. 31, Iss. 2 pp. 175-197. DOI: 10.1615/MultScienTechn.2019030437

23. Bubenhikov A.M., Kharlamov S.N. Matematicheskie modeli ne odnoprodnoy anizotropnoy turbulentnosti vo vnutrennikh techeniyakh [Mathematical models of heterogeneous anisotropic turbulence in internal flows]. Tomsk, Tomsk State University Publ., 2001. 448 p.

24. Petukhov B.S., Polyakov A.F. Teploobmen pri smeshannoy konvektsii [Heat exchange in mixed turbulent convection]. Moscow, Nauka Publ., 1986. 192 p.

25. Musket M. Fizicheskie osnovy tekhnologii dobychi nefti [Physical basis of oil production technology]. Moscow, Izhevsk, Institute of Computer Investigations Publ., 2004. 608 p.

26. Mirzadzhanzade F.Kh., Khasanov M.M., Bakhtizin R.N. Modelirovanie protsessov neftegazodobychy. Nelineynost, neravnovesnost, neopredelennost [Modeling of oil and gas production processes. Nonlinearity, nonequilibrium, uncertainty]. Moscow, Izhevsk, Institute of Computer Investigations Publ., 2004 $368 \mathrm{p}$.

27. Kharlamov S.N., Kudelin N.S., Dedeev P.O. Hydrodynamic, heat and acoustic processes modeling in transport of rheologically complex viscous media technology in pipelines. XVIII Interna- tional scientific symposium in honour of academician M.A. Usov. IOP Publishing, IOP conf. series: Earth and environmental science, 2014, vol. 21, no. 012040, pp. 1-7. DOI: 10.1088/1755$1315 / 21 / 1 / 012040$

28. Benjan A., Khair K.R. Heat and mass transfer by natural convection in a porous medium. International journal of heat and mass transfer, 1985, vol. 28, Iss. 5, pp. 909-918.

29. Hong J.T., Tien C.L., Kaviani M. Non-Darcian effects on vertical plate natural convection in porous media with high porosities. International journal of heat and mass transfer, 1985, vol. 28, Iss.12, pp. 2149-2159.

30. Dhananjay Yadav. Numerical examination of the thermal instability in an anisotropic porous medium layer subjected to rotation and variable gravity field. Special Topics \& Reviews in Porous Media: an International Journal, 2020, vol. 11, Iss. 1, pp. 395-407.

31. Larson R.E., Higdon J.J.L. Microscopic flow near the surface of two-dimensional porous media. Part.2 Transverse flow. Journal of Fluid Mechanics, 1987, vol. 178, pp. 119-136.

32. Gerschini G.Z., Zhukhovitskii E.M. Konvektivnaya ustoychivost neszhimaemoy zhidkosti [Convective stability of incompressible fluid]. Moscow, Nauka Publ., 1972. 392 p.

33. Obembe Abiola David, M. Enamul Hossain, Sidqi A. AbuKhamsin. Variable-order anomalous heat transport mathematical models in disordered and heterogeneous porous media. Second Thermal and Fluids Engineering Conference. Las Vegas, NV, USA, 2017, 2-5 April. pp. 2225-2239. DOI: 10.1615/ TFEC2017.prm.017616

34. Chetteti Ram Reddy, Padigepati Naveen, Darbhasayanam Srinivasacharya. Effects of nonlinear convection and cross-diffusion for the flow of darcy-forchheimer model micropolar fluid with convective boundary condition. Computational Thermal Sciences: an International Journal, 2019, vol. 11, Iss. 3, pp. 205-218. DOI: 10.1615/ComputThermalScien.2018019453

35. Vieiro J.J., Akhiiartdinov A., Sævik S., Larsen C.M., Nydal O.J. Two-way coupled fluid-structure interaction of gas-liquid slug flow in a flexible riser: small-scale experiments and simulations. Multiphase Science and Technology, 2019, vol. 31, Iss. 1, pp. $27-43$. DOI: 10.1615/MultScienTechn.2019029489

36. Saman Beyhaghi, Krishna Pillai. Estimation of tortuosity and effective diffusivity tensors using closure formulation in a sintered polymer wick during transport of a nondilute, multicomponent liquid mixture. Special Topics \& Reviews in Porous Media: an International Journal, 2011, vol. 2, Iss. 4, pp. 267-282. DOI: 10.1615/SpecialTopicsRevPorousMedia.v2.i4.20

37. Majid Molki. The swirling motion of dribbling honey. $5^{\text {th }}$ Thermal and Fluids Engineering Conference (TFEC). New Orleans, LA, USA, 2020, 5-8, April. pp. 321-332. DOI: 10.1615/TFEC2020. fnc. 031576

38. Kharlamov S.N. Characteristics of flow and heat transfer in a turbulent twisted flow. Heat Transfer Research, 2002, vol. 33, Iss. 1-2, pp. 64-74.

Received: 23 December 2021.

\section{Information about the authors}

Igor A. Melnik, Dr. Sc., professor, acting head of the department, National Research Tomsk Polytechnic University. Sergey N. Kharlamov, Dr. Sc., professor, National Research Tomsk Polytechnic University. 